\title{
Effect of EZH2 on pulmonary artery smooth muscle cell migration in pulmonary hypertension
}

\author{
YING WANG $^{1,2}$, XIAO-XI HUANG ${ }^{2,3}$, DONG LENG ${ }^{1,2}$, JI-FENG LI $^{2,4}$, YAN LIANG ${ }^{1,2}$ and TAO JIANG ${ }^{5,6}$ \\ ${ }^{1}$ Department of Clinical Laboratory, Beijing Chao-Yang Hospital, Capital Medical University; \\ ${ }^{2}$ Key Laboratory of Respiratory and Pulmonary Circulation Disorders, Institute of Respiratory Medicine; \\ ${ }^{3}$ Medical Research Center, Beijing Chao-Yang Hospital, Capital Medical University; \\ ${ }^{4}$ Department of Respiratory and Critical Care Medicine, Beijing Chao-Yang Hospital, Capital Medical University, \\ Beijing 100020; ${ }^{5}$ Department of Neurosurgery, Beijing Tiantan Hospital, Capital Medical University; \\ ${ }^{6}$ Beijing Neurosurgical Institute, Capital Medical University, Beijing 100070, P.R. China
}

Received December 5, 2019; Accepted October 27, 2020

DOI: $10.3892 / \mathrm{mmr} .2020 .11768$

\begin{abstract}
Pulmonary hypertension (PH) is a life-threatening disease that often involves vascular remodeling. Although pulmonary arterial smooth muscle cells (PASMCs) are the primary participants in vascular remodeling, their biological role is not entirely clear. The present study analyzed the role of enhancer of zeste homolog 2 (EZH2) in vascular remodeling of $\mathrm{PH}$ by investigating the behavior of PASMCs. The expression levels of EZH2 in PASMCs in chronic thromboembolic pulmonary hypertension (CTEPH), a type of $\mathrm{PH}$, were detected. The role of EZH2 in PASMC migration was investigated by wound-healing assay following overexpression and knockdown. Functional enrichment analysis of the whole-genome expression profiles of PASMCs with EZH2 overexpression was performed using an mRNA Human Gene Expression Microarray. Quantitative (q)PCR was performed to confirm the results of the microarray. EZH2 expression levels increased in CTEPH cell models. The overexpression of EZH2 enhanced PASMC migration compared with control conditions. Functional enrichment analysis of the differentially expressed genes following EZH2 overexpression indicated a strong link between EZH 2 and the immune inflammatory response and oxidoreductase activity in PASMCs. mRNA expression levels of superoxide dismutase 3 were verified by qPCR. The results suggested that EZH2 was involved in the migration of PASMCs in $\mathrm{PH}$, and may serve as a potential target for the treatment of $\mathrm{PH}$.
\end{abstract}

Correspondence to: Dr Tao Jiang, Department of Neurosurgery, Beijing Tiantan Hospital, Capital Medical University, 119 South Fourth Ring West Road, Fengtai, Beijing 100070, P.R. China E-mail: zacharytaojiang@163.com

Key words: enhancer of zeste homolog 2, hypoxic pulmonary arterial hypertension, chronic thromboembolic pulmonary hypertension, pulmonary artery smooth muscle cells, migration, functional enrichment analysis

\section{Introduction}

Pulmonary hypertension $(\mathrm{PH})$ is a progressive and life-threatening disease characterized by increased pulmonary artery pressure and pulmonary vascular resistance. PH can be divided into five clinical types according to different etiologies (1). However, different categories of PH share a common pathogenesis of vascular remodeling involving thickening of pulmonary vasculature and invasive proliferation of smooth muscle cells (2). Pulmonary smooth muscle cell (PASMC) proliferation and migration to the intima of pulmonary arteries serve a critical role in the process of pulmonary vascular remodeling in $\mathrm{PH}$ (3).

The underlying mechanism and genes associated with PASMC migration in PH have not been fully elucidated. Enhancer of zeste homolog 2 (EZH2), a histone methyltransferase highly expressed in multiple types of cancer, such as thyroid and prostate cancer $(4,5)$, promotes tumor cell proliferation and migration via epigenetic regulation of cancer-associated gene expression levels (6). EZH2 expression levels are also associated with tumor invasiveness (7). Considering that epigenetic alterations have been reported in certain types of $\mathrm{PH}$ that can be regarded as a cancer-like disease, EZH2 may be involved in the pulmonary arterial remodeling process in $\mathrm{PH}(8-10)$. EZH2 has been demonstrated to be associated with hypoxia-induced $\mathrm{PH}$ in mice and participates in the proliferation and migration of PASMCs (11). To the best of our knowledge, however, there is little evidence to demonstrate how EZH2 functions in the pathogenesis of PASMC migration in $\mathrm{PH}$.

Hypoxic pulmonary arterial hypertension (HPAH), a common type of $\mathrm{PH}$, is caused by high altitude or persistent hypoxic conditions induced by pulmonary disease, particularly chronic obstructive pulmonary disease (1). Previous studies on HPAH demonstrated that structural remodeling in pulmonary arteries contributes to its development and occur over a relatively short time period $(12,13)$. Chronic thromboembolic pulmonary hypertension (CTEPH) is another important form of PH characterized by one or multiple episodes of pulmonary embolism. Remodeling of distal pulmonary vessels following 
initial pulmonary thromboembolism may be responsible for the formation of CTEPH (14). The present study analyzed hypoxia-induced PASMCs and those isolated from the endarterectomized tissue of patients with CTEPH. In order to determine the role of EZH2 in $\mathrm{PH}$, the effect of EZH2 on PASMC migration was investigated using human CTEPH cell models. Pathways that contribute to this process following EZH2 overexpression were also identified.

The present results revealed high expression levels of EZH2 in hypoxia-induced and CTEPH PASMCs. Furthermore, EZH2 affected PASMC migration. The present study also reported genome-wide characteristics of EZH2 overexpression. These results may provide insight into the mechanism associated with PH artery remodeling.

\section{Materials and methods}

Ethics approval. The present study was approved by the Research Ethics Committee of the Beijing Chao-Yang Hospital of Capital Medical University. Written informed consent was provided by all patients before the procedure was initiated.

Cell preparation. Normal human PASMC line (N-PASMC; cat. no. 3110) was purchased from ScienCell Research Laboratories, Inc. and used for EZH2 transfection. Cells were cultured in smooth muscle cell growth medium (SMCM) (ScienCell Research Laboratories, Inc.), including smooth muscle basal medium, FBS (2\%), smooth muscle cell growth supplement $(1 \%)$, penicillin $(200 \mu \mathrm{g} / \mathrm{ml})$ and streptomycin (200 IU/ml). All cells were incubated in a 95\% humidified incubator at $37^{\circ} \mathrm{C}$ with $5 \% \mathrm{CO}_{2}$ and $21 \% \mathrm{O}_{2}$ and passaged after reaching $80-90 \%$ confluence.

A total of three consecutive patients with CTEPH diagnosed by pulmonary angiography and right heart catheterization at Beijing Chao-Yang Hospital (Beijing, China) between January 2013 and December 2014 were enrolled in the study. The patients included two male patients and one female patient, aged between 49 and 61 years. All patients diagnosed with CTEPH met the following criteria after 3 months of effective anticoagulation treatment: Mean pulmonary arterial pressure $\geq 25 \mathrm{mmHg}$ with normal wedge pressure $\leq 15 \mathrm{mmHg}$ and at least one pulmonary segmental perfusion defect revealed by lung perfusion scanning or pulmonary artery occlusion detected by multi-detector computed tomography angiography or pulmonary angiography. The World Health Organization (WHO) classification was used to classify patients (15). Exclusion criteria included ventriculo-atrial shunt, malignancies, and other lung diseases. Clinical data were recorded.

PASMCs from patients with CTEPH were isolated and cultured as previously described $(16,17)$. Briefly, pulmonary endarterectomy resection tissues from patients were cut into $1 \mathrm{~mm}^{3}$ pieces and then centrifuged at $300 \mathrm{x} \mathrm{g}$ for $5 \mathrm{~min}$ at room temperature. Following resuspension in SMCM, the resultant suspension was seeded in dishes and incubated in a $95 \%$ humidified incubator at $37^{\circ} \mathrm{C}$ with $5 \% \mathrm{CO}_{2}$ for 1 week until PASMCs were observed from adherent tissue pieces. The PASMC phenotypes of isolated cells were characterized by immunofluorescence using monoclonal antibodies against human $\alpha$-smooth muscle actin ( $\alpha$-SMA; 1:200; cat. no. ab124964; Abcam) and smooth muscle myosin heavy chain (1:100; cat. no. sc-6956; Santa Cruz Biotechnology, Inc.) respectively, for $1 \mathrm{~h}$ at room temperature. All cells were passaged after reaching $80-90 \%$ confluence prior to measurement of EZH2 expression levels.

Transient transfection of PASMCs. For EZH2 overexpression, the EZH2 gene was subcloned into the pEZ-M98 vector (GeneCopoeia, Inc.) to generate pM-EZH2. Cultured N-PASMCs were then transfected with pM-EZH2 vector using the Neon Transfection System (MPK5000) from Invitrogen (Thermo Fisher Scientific, Inc.), according to the supplier's instructions. Briefly, cells were cultured in $60-\mathrm{mm}$ dishes to $70 \%$ confluency and then transfected with $2 \mu \mathrm{g}$ plasmid DNA $(1,375 \mathrm{~V} ; 20 \mathrm{~ms})$ at room temperature. Empty vector pEZ-M98 (pM) was used as a transfection control.

For EZH2 knockdown, PASMCs from CTEPH patient 3 were cultured in 60-mm dishes and transfected with 160 pmol small interfering (si)RNA (Shanghai GenePharma Co., Ltd.) and treated with Lipofectamine RNAiMAX reagent from Invitrogen (Thermo Fisher Scientific, Inc.) at room temperature, according to the manufacturer's instructions. The sequences were sense, 5'-CCUGACCUCUGUCUUACU UTT-3' and antisense, 5'-AAGUAAGACAGAGGUCAG GTT-3'. RNAi negative control that had no homology with mammalian genes was used as the transfection control. The RNAi negative control sequences were sense, 5'-UUCUCC GAACGUGUCACGUTT-3' and antisense, 5'-ACGUGACAC GUUCGGAGAATT-3'. After 48 h, transfection efficiency was determined by both PCR and western blot analysis for EZH2.

Wound healing assay. At $12 \mathrm{~h}$ after transfection, the transfection mixture was replaced with $0.2 \% \mathrm{FBS}$, and a straight scratch was created using a $200-\mu 1$ pipette tip on a monolayer of confluent PASMCs. Images were captured at 0 and $12 \mathrm{~h}$ after scratching to visualize migrated cells and wound healing using phase microscopy (magnification, $x 40$ ). The distance of cell movement from the wound edge into the wound area indicated the extent of cell migration. A total of 10 points, evenly spaced, were selected on each edge, and the minimum distance to the scratch edge was measured. The cell migration rate was calculated with the following formula: [Scratch width $(0 \mathrm{~h})$-scratch width $(12 \mathrm{~h})] / \mathrm{scratch}$ width $(0 \mathrm{~h}) \times 100 \%$. The experiment was repeated three times, and the average value was calculated.

Gene microarray analysis. In order to evaluate the effect of EZH2 on PASMCs, total RNA was isolated using TRIzol (Invitrogen; Thermo Fisher Scientific, Inc.) following EZH2 overexpression. mRNA expression levels were profiled using mRNA + IncRNA Human Gene Expression Microarray V4.0 (CapitalBio Corporation) according to the manufacturer's instructions. For microarray analysis, Agilent Feature Extraction (V10.7; Agilent Technologies, Inc.) was used for data extraction and quantification. Then, raw data were summarized and normalized at the transcript level using the GeneSpring GX program (V12.0; Agilent Technologies, Inc.). The unpaired t-test was applied to filter genes with differential expression in the control vs. experimental groups. Fold-change in differentially expressed mRNAs $>2.0$ and 
$\mathrm{P}<0.05$ were considered to indicate a statistically significant difference between experimental and control groups. Certain differentially expressed genes were then validated by reverse transcription-quantitative (RT-q)PCR.

Gene Ontology (GO) and pathway functional enrichment analysis. Following microarray screening for mRNAs from PASMCs transfected with pM-EZH2 and controls, the differentially expressed genes were grouped into functional categories by performing $\mathrm{GO}$ enrichment analysis according to the GO database (geneontology.org/), which categorizes genes into regulatory networks on the basis of 'Biological Process', 'Molecular Function' and 'Cellular Function'. The P-value of the significance level for each gene with differential expression was estimated with Fisher's exact test. $\mathrm{P}<0.05$ was considered to indicate a statistically significant difference.

Pathway enrichment analysis was performed using the Kyoto Encyclopedia of Genes and Genomes (KEGG) (18), BioCarta (19) and Reactome databases (20) with KOBAS software (version 3.0) (21). Significant enrichments were calculated and filtered by $\mathrm{P}<0.05$.

$R T$-qPCR . Relative expression levels of EZH2, superoxide dismutase 3 (SOD3) and NADPH oxidase 1 (NOX1) were determined by RT-qPCR analysis. Total RNA was isolated from PASMCs with EZH2 transfection using TRIzol ${ }^{\circledR}$ (Invitrogen; Thermo Fisher Scientific, Inc.). RT was conducted using a ReverTra Ace qPCR kit (Toyobo Life Science). Briefly, $0.5 \mu \mathrm{g}$ total RNA was first reverse transcribed using reverse transcriptase Mix at the following conditions: $37^{\circ} \mathrm{C}$ for $15 \mathrm{~min}$ and $98^{\circ} \mathrm{C}$ for $5 \mathrm{~min} ; 4^{\circ} \mathrm{C}$. qPCR quantification was performed as previously described (15). All experiments were performed in triplicate. The primer sequences were: EZH2 forward, 5'-AAT CATGGGCCAGACTGGGAAGAA-3' and reverse, 5'-TCT TGAGCTGTCTCAGTCGCATGT-3'; SOD3 forward, 5'-GGC CTCCATTTGTACCGAAA-3' and reverse, 5'-AGGGTCTGG GTGGAAAGGT-3'; NOX1 forward, 5'-CCCCAAGTCTGT AGTGGGAGTT-3' and reverse, 5'-CGCAGGCTCTTTGCC AAA-3'; and GAPDH forward, 5'-TGACTTCAACAGCGA CACCCA-3' and reverse, 5'-CACCCTGTTGCTGTAGCC AAA-3'. Human GAPDH was used as an endogenous control to normalize gene expression levels.

Statistical analysis. All experiments were performed in triplicate. Data are presented as the mean \pm SD. Data were analyzed using SPSS software (version 13.0; SPSS, Inc.) Statistical significance was determined using an unpaired Student's t-test. $\mathrm{P}<0.05$ was considered to indicate a statistically significant difference.

\section{Results}

Increased EZH2 expression levels in PASMCs derived from patients with CTEPH. In order to analyze EZH2 expression levels, PASMCs were isolated from tissue obtained during endarterectomy from 3 patients with CTEPH. The clinical information for patients is summarized in Table SI. The mRNA expression levels of EZH2 were assessed in PASMCs from CTEPH patients. EZH2 mRNA expression levels were significantly increased in PASMCs from CTEPH patients,

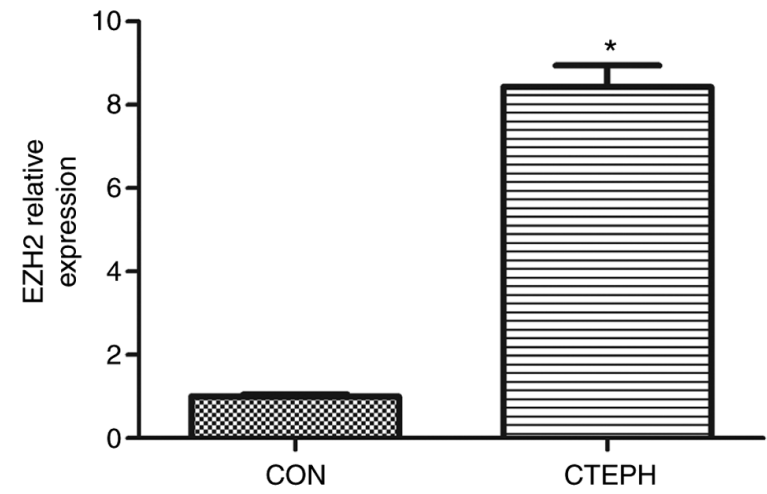

Figure 1. Relative expression levels of EZH2. The relative expression levels of EZH2 in normal control PASMCs and PASMCs from CTEPH patient 3 were assayed by reverse transcription-quantitative PCR. " $\mathrm{P}<0.05$ vs. CON. EZH2, enhancer of zeste homolog 2; PASMC, pulmonary arterial smooth muscle cell; CTEPH, chronic thromboembolic pulmonary hypertension; CON, control.

compared with control PASMCs (Fig. S1). PASMCs from CTEPH patient 3 exhibited the strongest EZH2 expression levels among the patients (Fig. 1 and Table SII).

EZH2 affects PASMC migration. EZH2 expression levels were determined by western blot analysis following overexpression by pM-EZH2 vector and knockdown by siRNA (Fig. S2). The role of the EZH2 gene in affecting PASMC migration was then investigated by performing a wound healing assay. Following 12-h wounding, the gap scratched by a pipette tip was filled with more cells in the EZH2-overexpression group compared with the control. However, the distance of the scratch between the migration edges of the cells was significantly wider in the EZH2 interference group, compared with the control. The migration distance of N-PASMCs following EZH2 transfection was increased compared with that observed for the control. Silencing of EZH2 by siRNA significantly inhibited the migration of PASMCs derived from patients with CTEPH (Figs. 2 and 3). This indicated that EZH2 increased the migration of PASMCs and participated in the pathogenesis of PAH.

EZH2-associated differences in gene expression levels. N-PASMCs transfected with EZH2 and control were collected and microarray analysis was used to investigate changes in gene expression levels. A data set from the microarray was subjected to supervised hierarchical clustering analysis. Samples were divided into two groups based on differential expression levels of genes (Fig. 4). A total of 192 genes with statistically significant changes were detected. Among these, 122 genes were upregulated and 70 were downregulated following EZH2 transfection. The top 10 significantly up- or downregulated genes, including oncostatin M (OSM), Nod-like receptor (NLR)P12, IL-31, serum amyloid A2 (SAA2) and arachidonate 5-lipoxygenase activating protein (ALOX5AP), according to P-value are summarized in Table I.

GO and KEGG functional enrichment analysis reveals different genes are involved in inflammatory and immune processes. GO analysis of Cell Component revealed that differentially expressed genes were primarily enriched in 
A

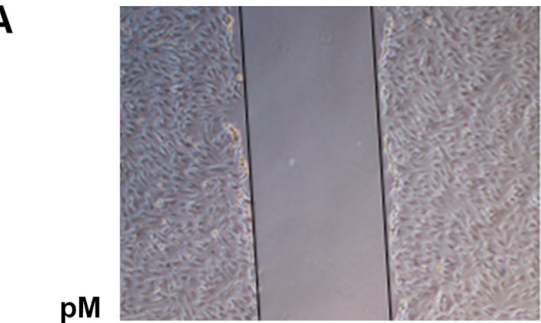

pM-EZH2

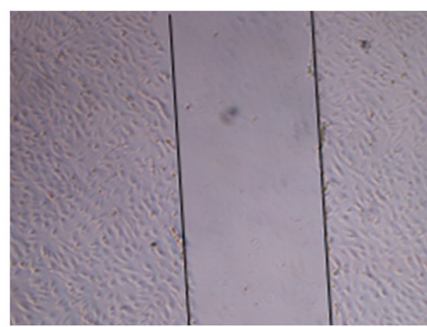

$\mathrm{Oh}$
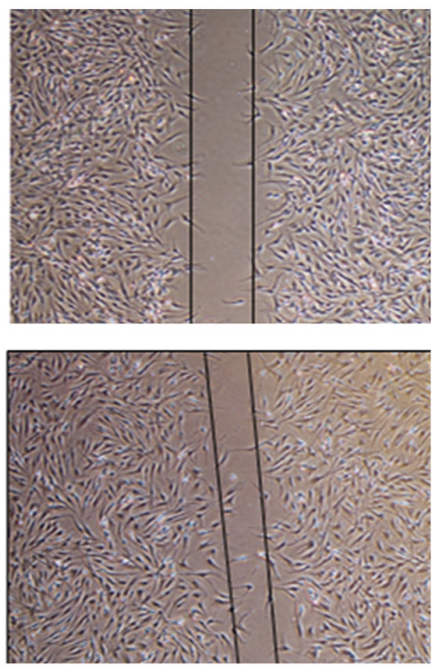

$12 \mathrm{~h}$
B

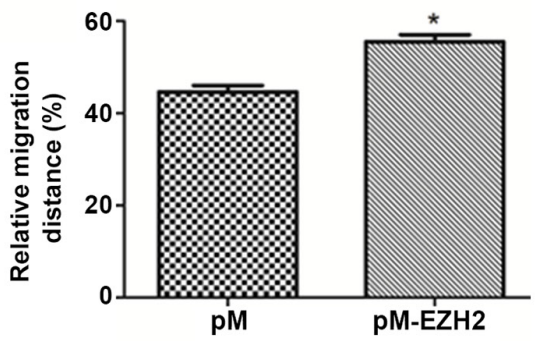

Figure 2. EZH2 overexpression increases PASMC migration during wound healing. N-PASMCs were transfected with plasmid pEZ-M98-EZH2 (pM-EZH2) or pEZ-M98 (pM) plasmid only (control). A scratch was created using a $200-\mu 1$ pipette tip on a confluent monolayer of cells. Cell migration distance was assayed as the distance from wound edges to the wound area. (A) Images obtained at 0 and $12 \mathrm{~h}$ in the pM-EZH2 and control groups. (B) Rate of cell migration in each group. ${ }^{*} \mathrm{P}<0.05$ vs. pM. EZH2, enhancer of zeste homolog 2; PASMC, pulmonary arterial smooth muscle cell; $\mathrm{N}$, normal.

A

si-NC
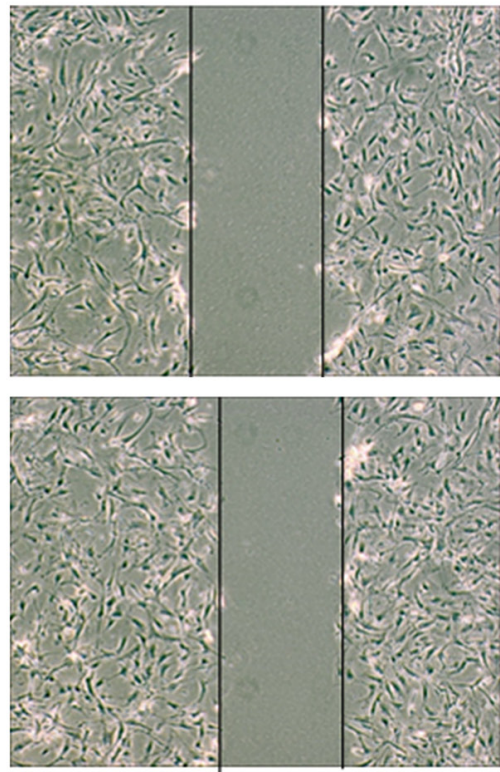
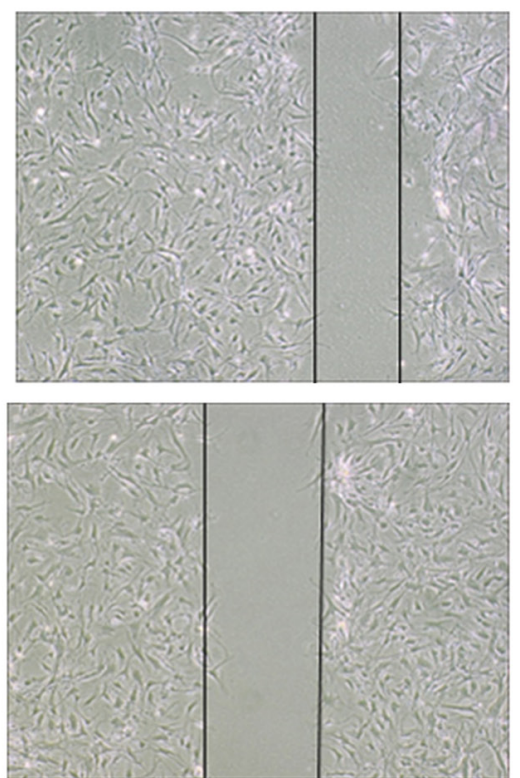

B

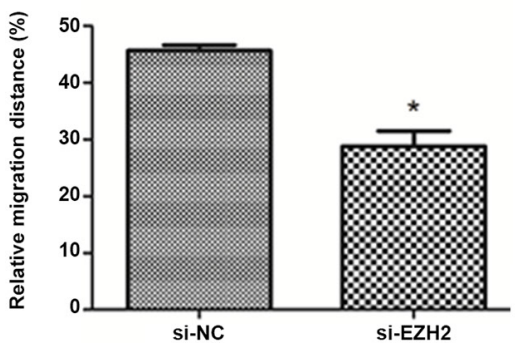

Figure 3. EZH2 interference inhibits CTEPH PASMC migration during wound healing. PASMCs from CTEPH Patient 3 were transfected with siRNA (si-EZH2). Scrambled siRNA was used as the RNA interference NC (si-NC). A scratch was created using a 200- $\mu 1$ pipette tip on a confluent monolayer of cells. Cell migration distance was measured as the distance from wound edges to the wound area. (A) Images obtained at 0 and $12 \mathrm{~h}$ after wounding in the si-EZH2 and control groups. (B) Cell migration rate in each group. ${ }^{*} \mathrm{P}<0.05$ vs. si-NC. EZH2, enhancer of zeste homolog 2; CTEPH, chronic thromboembolic pulmonary hypertension; PAMSC, pulmonary arterial smooth muscle cell; si, small interfering; NC, negative control.

'membranous components' (data not shown). The GO analysis of Molecular Function revealed differentially expressed genes associated with 'hydrogen ion channel activity' and 'oxidoreductase activity' (data not shown). GO analysis of 'Biological Process', as shown in Table II, revealed a strong signature for 'cellular response to $\mathrm{pH}$ ', 'inflammation and the immune response' related functions. Of the top 20 significant GO terms, 7 (35\%) were concerned with 'cellular response to $\mathrm{pH}$ ' or 'response to metal ion'; 8 (40\%) were involved in 'regulation of interleukin-6 biosynthetic process', 'interleukin-6 biosynthetic process', 'negative regulation of interleukin-6 biosynthetic process', 'immune system process' and 'humoral immune response mediated by circulating immunoglobulin' (Table II). NLRP12, toll-like receptor 9, complement C1q (C1Q)B and C chain were markedly enriched in these GO terms.

Pathway analysis of differentially expressed genes also implicated inflammation and the immune response-related pathways were involved in PASMCs migration mediated by EZH2. The most significant pathway was 'classical antibody-mediated complement activation' ( $\mathrm{P}=0.001)$. The second most significant pathway, 'Creation of C4 and C2 activators', 


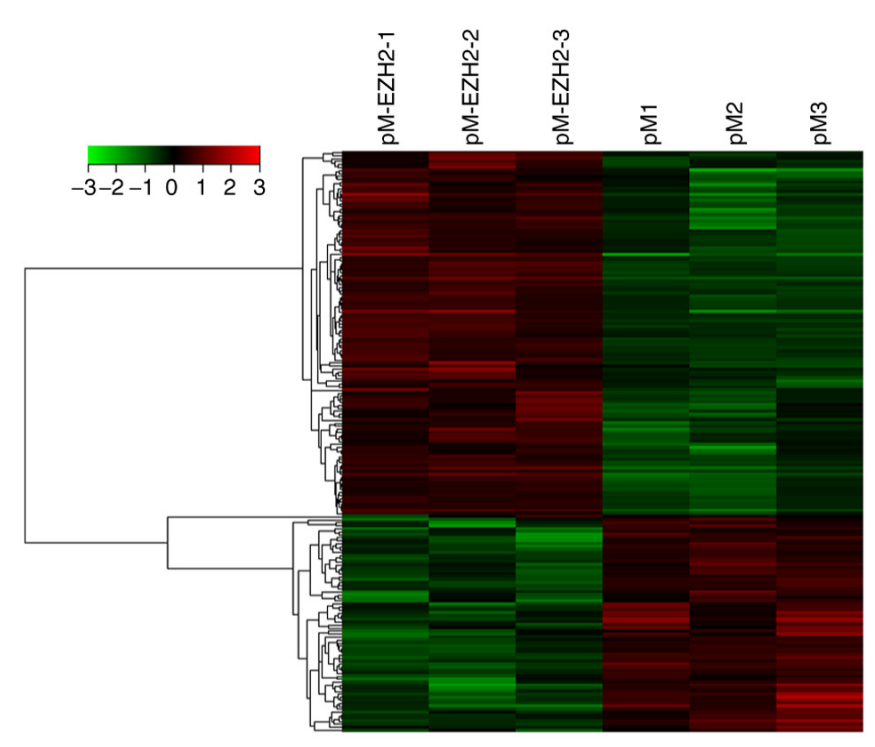

Figure 4. Supervised hierarchical cluster analysis of significantly differentially regulated genes from EZH2 overexpression and control pulmonary arterial smooth muscle cells. Rows represent the differentially expressed genes; columns represent the samples. Gene expression levels are depicted according to the color scale. Color change from green to red indicates a change from down- to upregulation. EZH2, enhancer of zeste homolog 2.

and fourth most significant pathway, 'Initial triggering of complement', were also associated with complement-associated pathways $(\mathrm{P}=0.03$ and 0.09 , respectively). The top 10 significant pathways included inflammatory and immune response mechanisms such as 'phosphorylation of CD3 and TCR $\zeta$ chains', 'synthesis of leukotrienes (LT) and eoxins (EX)' and the 'cytochrome P450-arranged by substrate type' (Fig. 5). The signature genes that regulated inflammatory and immune response processes included C1QC, CYP4F8 and ALOX5AP.

Validation of gene expression levels. SOD3 and NOX1 were selected for validation of mRNA expression levels via qPCR because functional analysis revealed that both genes were significantly enriched following EZH2 transfection. qPCR demonstrated that the mRNA expression level of SOD3 was decreased compared with the control (Fig. 6 and Table SIII), confirming the results of the microarray detection. However, NOX1 expression levels were not significantly altered.

\section{Discussion}

PASMCs are primary mediators of small pulmonary arterial remodeling, which is an important pathological process in the progression of $\mathrm{PH}$. The proliferation and migration of PASMCs serve key roles in this process (22). Epigenetic changes are involved in the development of certain types of PH (10). Furthermore, our laboratory previously revealed that DNA methylation changes in PASMCs are associated with the pathogenesis of CTEPH $(10,15)$. In the present study, upregulation of EZH2 was observed in PASMCs from patients with $\mathrm{CTEPH}$ and those induced by hypoxic conditions. Moreover, EZH2 affected the migration ability of N-PASMCs. Gene chips were used to identify the mechanism following EZH2
Table I. Top 10 significantly altered genes.

A, Upregulated genes

\begin{tabular}{lcc}
\hline Gene symbol & P-value & Fold-change \\
\hline OSM & 0.001 & 6.015 \\
FSHR & 0.009 & 4.793 \\
NLRP12 & 0.008 & 4.294 \\
LOC100996286 & $<0.001$ & 4.010 \\
LOC100131907 & $<0.001$ & 3.969 \\
CFAP61 & 0.001 & 3.876 \\
IL31 & 0.016 & 2.953 \\
SLC40A1 & 0.042 & 2.819 \\
CRX & 0.013 & 2.809 \\
lnc-DFNA5-3 & 0.006 & 2.675 \\
\hline
\end{tabular}

B, Downregulated genes

\begin{tabular}{lcc}
\hline Gene symbol & P-value & Fold-change \\
\hline SAA2 & 0.013 & 4.615 \\
C3orf67 & 0.015 & 4.593 \\
ABCC6 & 0.013 & 3.744 \\
SLC38A4 & 0.040 & 3.404 \\
TTN & 0.009 & 3.349 \\
ALOX5AP & 0.047 & 3.161 \\
LOC101929689 & 0.001 & 3.132 \\
C1orf229 & 0.011 & 3.132 \\
AP1S2 & 0.001 & 3.025 \\
lnc-CBWD5-1 & 0.010 & 3.022 \\
\hline
\end{tabular}

overexpression in N-PASMCs. Functional enrichment analysis revealed that pathophysiological pathways that participated in inflammatory and immune processes were controlled by EZH2-associated genes. The present study provides further understanding into vascular remodeling mechanisms underlying $\mathrm{PH}$.

EZH2 is a conserved methyltransferase of histone H3K27, which promotes cell proliferation, migration and invasiveness in tumorigenesis via epigenetic modifications $(23,24)$. HPAH and CTEPH are two common types of PH (1). PASMCs in HPAH and CTEPH exhibit proliferation and migration phenotypes similar to those of cancer cells $(25,26)$. In the present study, EZH2 expression levels were increased in both hypoxia-induced and patient-derived PASMCs, which is consistent with a previous study in a hypoxia-induced PAH mouse model (11). However, unlike CTEPH, HPAH is rarely treated with surgery, thus tissue samples from patients with HPAH are difficult to obtain. Hypoxia-induced human PASMCs were therefore used instead of HPAH tissue samples for the measurement of EZH2 mRNA expression levels. The present study did not investigate EZH2 mRNA expression levels in HPAH and only focused on CTEPH because EZH2 mRNA levels could not be measured in cells from $\mathrm{HPAH}$ tissue. Increased migration ability of N-PASMCs 
Table II. Top 20 significant GO terms of differential genes.

\begin{tabular}{|c|c|c|}
\hline GO term & Genes & P-value \\
\hline Cellular response to $\mathrm{pH}$ & HVCN1, KD1L3, NOX1 & 0.0002 \\
\hline Response to metal ion & $\begin{array}{l}\text { TTN, SLC18A2, SOD3, HVCN1, CYP11B1, } \\
\text { SLC40A1, ZACN, ALOX5AP }\end{array}$ & 0.0003 \\
\hline Negative regulation of interleukin-6 biosynthetic process & PRG4, NLRP12 & 0.0008 \\
\hline Regulation of interleukin-18 production & TLR9, NLRP12 & 0.0008 \\
\hline Response to transition metal nanoparticle & HVCN1, ZACN, SLC18A2, SOD3, SLC40A1 & 0.0008 \\
\hline Negative regulation of interleukin-6 production & PRG4, NLRP12, TLR9 & 0.0011 \\
\hline Interleukin-18 production & TLR9, NLRP12 & 0.0011 \\
\hline Response to $\mathrm{pH}$ & HVCN1, PKD1L3, NOX1 & 0.0012 \\
\hline Cellular response to acidic $\mathrm{pH}$ & PKD1L3, NOX1 & 0.0017 \\
\hline Positive regulation of response to wounding & TLR9, OSM, NLRP12, ANO6, CPB2 & 0.0021 \\
\hline Response to zinc ion & HVCN1, ZACN, SLC18A2 & 0.0025 \\
\hline $\begin{array}{l}\text { Humoral immune response mediated by circulating } \\
\text { immunoglobulin }\end{array}$ & C1QC, C1QB, HLA & 0.0032 \\
\hline Response to acidic $\mathrm{pH}$ & PKD1L3, NOX1 & 0.0039 \\
\hline Mitotic chromosome condensation & TTN, CDCA5 & 0.0039 \\
\hline Response to inorganic substance & $\begin{array}{l}\text { TTN, SLC18A2, SOD3, HVCN1, CYP11B1, } \\
\text { SLC40A1, ZACN, ALOX5AP }\end{array}$ & 0.0039 \\
\hline Immune system process & $\begin{array}{l}\text { PSMB11, RAB17, SLC7A10, PRG4, ANO6, } \\
\text { PRKCG, CYP11B1, SLC40A1, INPP4B, IGSF6, } \\
\text { C1QC, OSM, C1QB, TLR9, CD160, PYDC1, } \\
\text { DPP4, HLA, MLF1, GCSAM, CCL3L3, IL31 }\end{array}$ & 0.0046 \\
\hline Regulation of interleukin- 6 biosynthetic process & PRG4, NLRP12 & 0.0051 \\
\hline Cellular response to metal ion & HVCN1, ALOX5AP, CYP11B1, SLC40A1 & 0.0051 \\
\hline Photoreceptor cell differentiation & CEP290, CRX, MYO7A & 0.0053 \\
\hline Interleukin- 6 biosynthetic process & PRG4, NLRP12 & 0.0062 \\
\hline
\end{tabular}

GO, Gene Ontology.

overexpressing EZH2 and decreased migration ability of patient-derived PASMCs following EZH2 knockdown were confirmed, which was in line with previously reported changes in hypoxia-induced HPASMCs and cancer cells $(11,27)$. Collectively, the present findings indicated that EZH2 may serve a role in the migration of PASMCs in $\mathrm{PH}$ vascular remodeling. However, no changes in the proliferation or apoptosis of PASMCs following EZH2 transfection (data not shown) were detected in the present study, which was inconsistent with findings previously reported by Aljubran et al (11). It was speculated that different PASMC phenotypes may partly explain this difference: Previous studies have demonstrated that there may be multiple phenotypically distinct SMC populations in pulmonary arteries, and these distinct populations may serve different functions $(28,29)$.

EZH2-associated changes in gene expression levels and pathophysiological signaling pathways were investigated in the present study. The results identified a number of candidate genes, as well as pathophysiological pathways targeted by oxidoreductase activity, inflammation and immune processes, that provide a basis for further investigation of the mechanism of EZH2 in $\mathrm{PH}$ vascular remodeling. The present results revealed that three of the top 10 significantly upregulated genes (OSM, NLRP12 and IL-31) were closely associated with inflammatory and immune responses. As the most differentially upregulated gene, OSM is a secreted member of the IL- 6 family and serves an important role in maintaining homeostasis of the internal environment under chronic inflammation (30). NLRP12 belongs to non-classical NLRP molecules and has been reported to regulate inflammation and tumorigenesis $(31,32)$. IL-31 is a cytokine belonging to the IL-6 family and shares a common signaling receptor subunit with OSM (33). IL-6 has been reported to be involved in airway inflammation and remodeling in vascular remodeling $(34,35)$. As is true for a number of proteins in the IL-6 family, the roles of IL-31 and OSM in vascular remodeling remain unclear. The microarray analysis identified SAA2 and ALOX5AP among the 10 most downregulated genes. This result appeared to contradict the proinflammatory roles of SAA2 and ALOX5AP reported in previous studies, which have established that SAA is expressed at increased levels in numerous inflammatory conditions, including trauma, infection and tumor growth, and secreted by the liver as an acute-phase protein (36-38). However, the potential roles of local SAA variants remain to be elucidated. De Buck et al (39) described the extrahepatic production of 
Top10 significant pathways of differential genes

$(-\mathrm{LgP})$

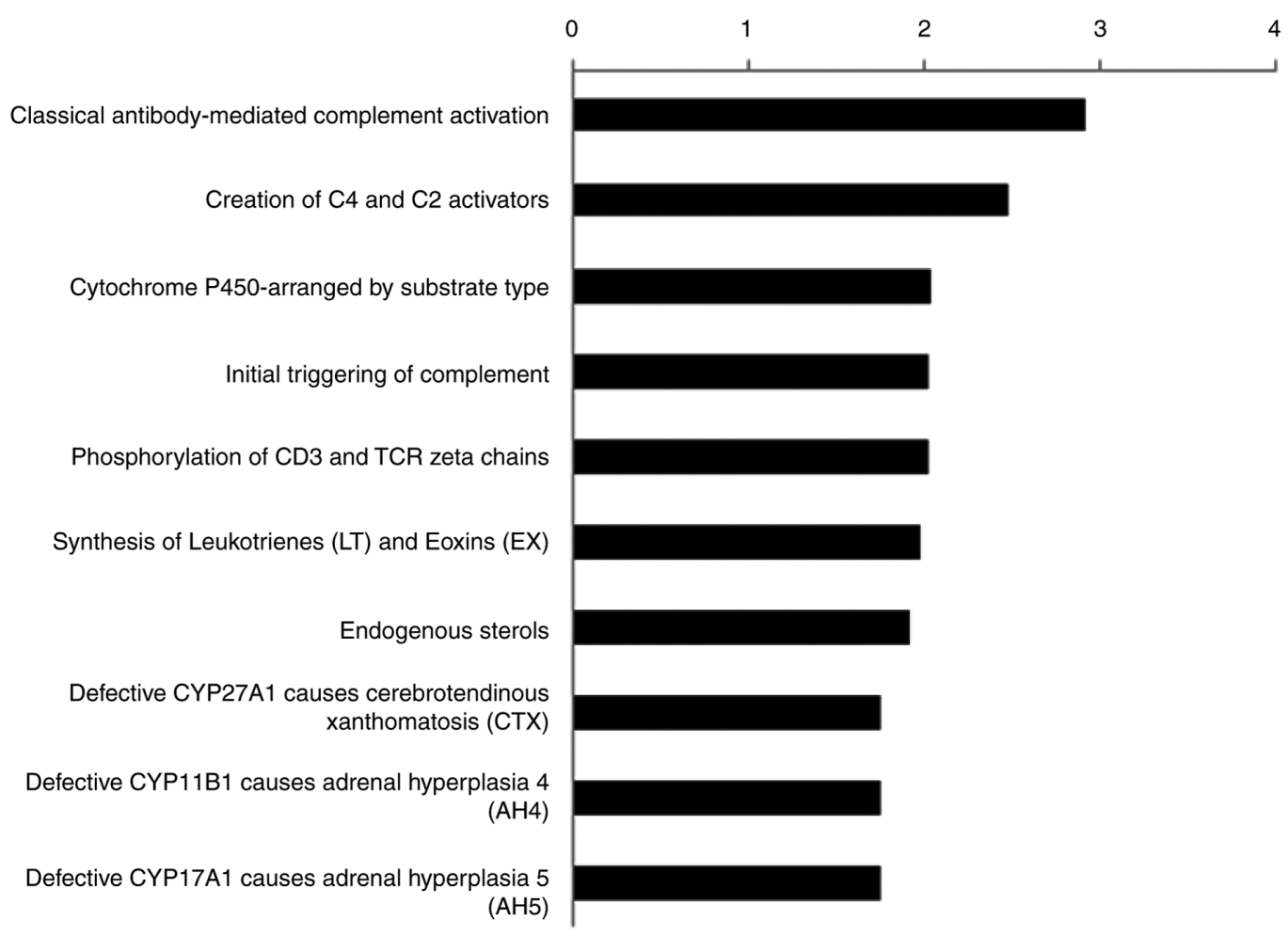

Figure 5. Top 10 pathways with significant differences in gene expression levels. The criterion for significance was $\mathrm{P}<0.05$. LgP ( $\mathrm{x}$-axis) represents the significance level. TCR, T-cell receptor.

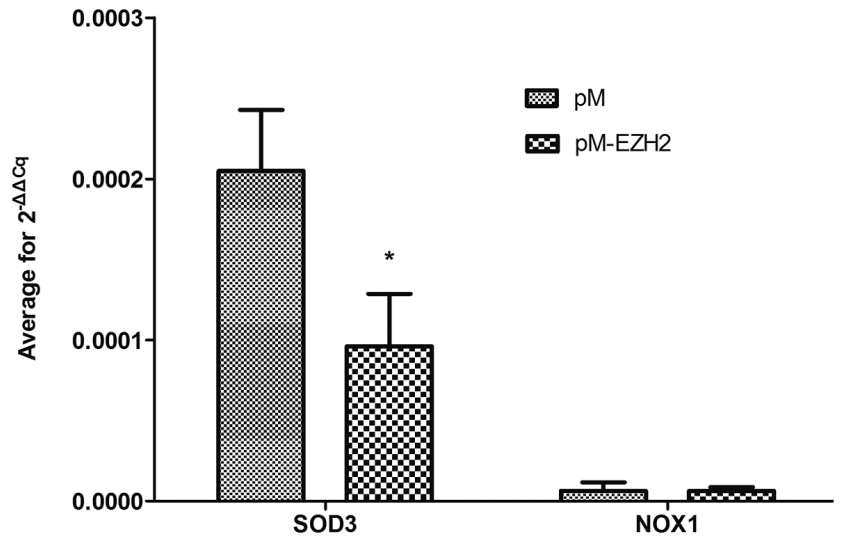

Figure 6. Validation of array data. Normal pulmonary arterial smooth muscle cells were transfected with plasmid pM-EZH2 or pM plasmid only (control). Expression levels of SOD3 and NOX1 $(n=3)$ were assayed by reverse transcription-quantitative PCR. ${ }^{*} \mathrm{P}<0.05$ vs. pM. EZH2, enhancer of zeste homolog 2; SOD3, superoxide dismutase 3; NOX1, NADPH oxidase 1.

SAA variants by a number of types of tissues, such as brain and colon tissues, with differential expression levels of SAA1 and SAA2. Jumeau et al (40) also identified SAA1 as an important SAA acute-phase gene induced in human monocytes and derived macrophages in the presence of stimulants. The ALOX5AP gene is required for LT synthesis, which is involved in numerous types of inflammatory response (37). The present microarray analysis revealed downregulated expression levels of ALOX5AP $(\mathrm{P}=0.047)$. As demonstrated by Wang et al (41), microarrays may not provide accurate results when used to measure differential gene expression at levels near the threshold for detection. No significant change in the expression levels of ALOX5AP was detected (data not shown). Therefore, results reported in relation to ALOX5AP expression levels require further study.

The present results demonstrated significant downregulation of SOD3 levels. In 2010, Archer et al (9) confirmed that deficiency of SOD2 causes PAH by impairing redox signaling and inducing PASMC phenotype transition, which is highly associated with epigenetic regulation by EZH2. Soon et al (42) also demonstrated that BMPR-II deficiency instigates the development of PAH by decreasing SOD3 expression levels and exaggerating the inflammatory response both in vitro and in vivo. Therefore, the role of EZH2 in PASMC migration may be associated with redox and inflammation reactions.

GO term enrichment analysis confirmed the aforementioned observations. GO analysis revealed immune system processes that involve IL- $6,-18$ and -1 and $N F-\kappa B$ regulation and complement activation predominated. Increasing evidence has confirmed that these inflammatory factors promote vascular remodeling in $\mathrm{PH}$ (43-45). Significantly enriched GO terms also included cellular response to hydrogen potential, $\mathrm{pH}$ and oxidoreductase activity, which are associated with abnormal energy metabolism in mitochondria, reactive oxygen species (ROS) and an acidic microenvironment. A potential mechanism of cancer-like abnormalities such as a PASMC phenotype shift in PH may be mitochondrial disorder and glycolysis, which is known as the Warburg phenotype in tumors (46-48). In PAH and cancer, abnormal mitochondrial metabolism and redox signaling lead to decreased ROS levels 


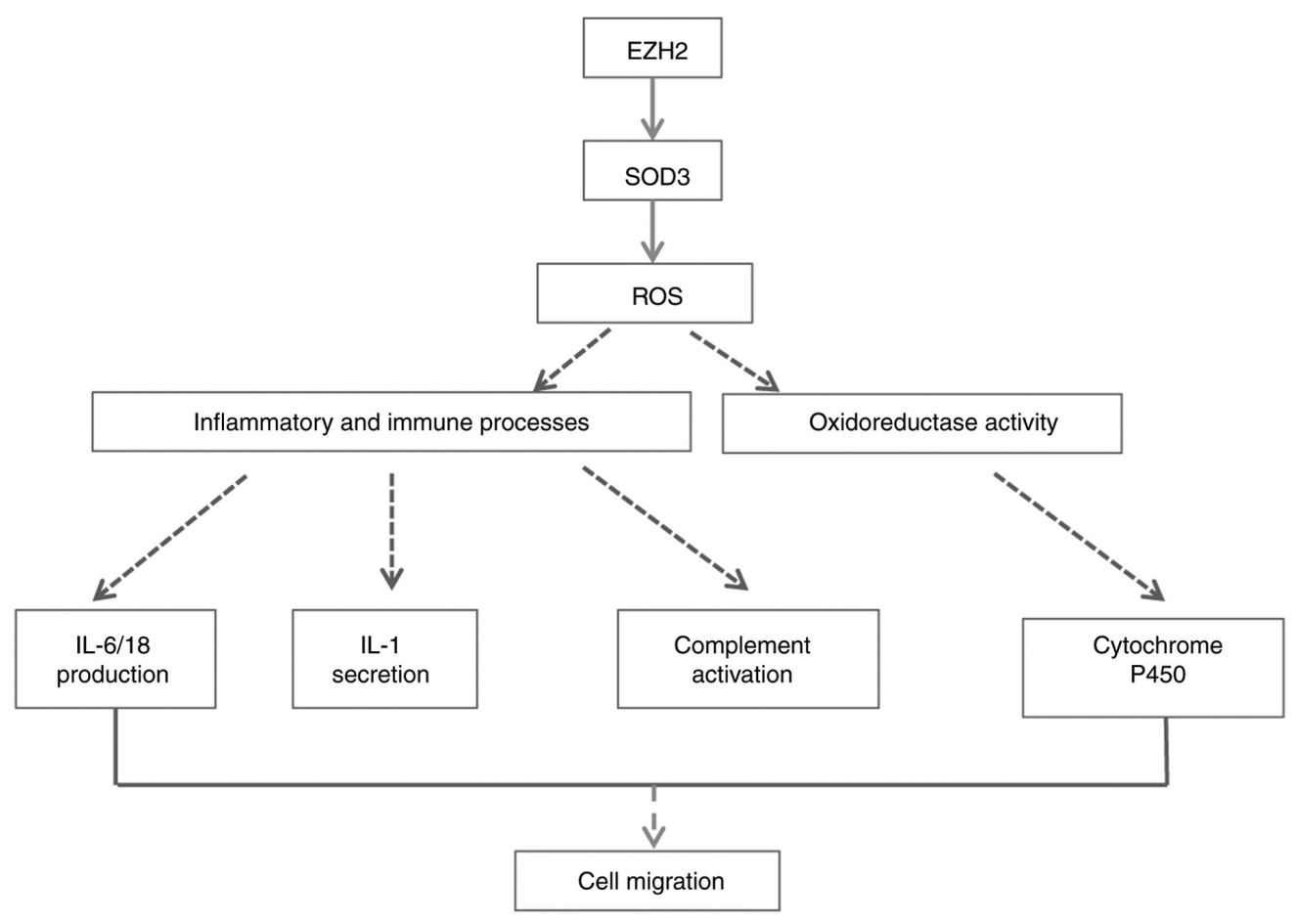

Figure 7. Potential mechanism for EZH2 modulation. EZH2, enhancer of zeste homolog 2; SOD3, superoxide dismutase 3; ROS, reactive oxygen species.

and a shift from oxidative to glycolytic metabolism (49). There is a close association between ROS and the occurrence and development of inflammation (50). Sutendra et al (51) reported that abnormalities of mitochondrial metabolism and decreased ROS levels are linked to the chronic inflammatory process in PAH and progressive expansion of SMC-like cells that obliterate the vascular lumen. SOD has been recognized as a redox-signaling molecule that regulates pulmonary vascular tone and structure and may have therapeutic potential in inflammation (52). Soon et al (42) reported that SOD3 loss is necessary for the proinflammatory phenotype in PAH. The aforementioned studies suggest that there may be an important interplay between ROS and inflammation in SMC. Therefore, we hypothesize that EZH2 may lead to epigenetic repression of SOD3, thus promoting PASMC migration in PAH via inflammatory and immune processes. This chain of events is consistent with the pathogenic mechanism of PAH (53).

Further pathway analysis supported these inflammatory changes, including effects on CD3 and TCR $\zeta$ chains and LT synthesis. Pathway analysis also identified a role for complement-associated pathways in the EZH2-mediated migration of PASMCs. A previous study revealed that $\mathrm{C} 3$ complement contributes to the development of hypoxia-induced $\mathrm{PH}$ in mice (54), and this process is associated with pulmonary vascular remodeling. C3 complement levels are significantly altered in patients with idiopathic PAH (55). In CTEPH, the inflammatory response has also been revealed to play a critical role (56-58).

A total of two genes significantly enriched in GO terms and pathways were selected to verify mRNA expression levels. The decreased expression levels of SOD3 were consistent with the microarray results. Potential mechanism pathways are presented in Fig. 7. The mechanisms of the aforementioned genes and clear functions modulated by EZH2 in PH should be investigated in further studies.

There are limitations of the present study. First, increased PASMC migration was detected following EZH2 overexpression. Since increased SMC migration is a feature of the synthetic phenotype, the expression levels of synthetic phenotype markers should be assessed to determine the effects of EZH2 overexpression on the PASMC phenotype. Second, these findings indicated that EZH2 is increased in PASMC models of $\mathrm{PH}$ and provide a potential preliminary mechanism for the effects of EZH2 on PASMC migration in PH. However, the exact molecular mechanism requires further investigation. For example, T-cell coculture experiments should be performed to validate our finding that EZH2 may regulate inflammatory and immune processes. Third, the present findings are also limited by cell type, since there are distinct SMC populations in pulmonary arteries that may serve different functions. Further validation should be performed using different cells and patient-derived cell lines.

In conclusion, the role of histone methyltransferase EZH2 in $\mathrm{PH}$ was investigated. The results indicated that EZH2 promoted human PASMC migration and was increased in CTEPH PASMCs. The enrichment functions affected by EZH2 focused on inflammatory and immune processes. qPCR confirmed significantly altered gene expression levels of SOD3, which suggested an association between EZH2, SOD3, PASMC migration and inflammatory and immune processes in PAH. The present findings may aid the search for therapeutic targets for $\mathrm{PH}$ and further investigation on $\mathrm{EZH} 2$ in $\mathrm{PH}$ is warranted.

\section{Acknowledgements}

Not applicable. 


\section{Funding}

The present study was supported by Capital's Funds for Health Improvement and Research (grant no. CFH 2018-2-2042).

\section{Availability of data and materials}

The datasets used and/or analyzed during the current study are available from the corresponding author on reasonable request.

\section{Authors' contributions}

YW, XXH and DL performed the experiments. YW drafted the manuscript. JFL analyzed the data. YL analyzed and interpreted the data. TJ directed and designed the study. All authors read and approved the final manuscript.

\section{Ethics approval and consent to participate}

The present study was approved by the Research Ethics Committee of the Beijing Chao-Yang Hospital of Capital Medical University. Written informed consent was provided by all patients before the procedure was initiated.

\section{Patient consent for publication}

Not applicable.

\section{Competing interests}

The authors declare that they have no competing interests.

\section{References}

1. Simonneau G, Gatzoulis MA, Adatia I, Celermajer D, Denton C, Ghofrani A, Gomez Sanchez MA, Krishna Kumar R, Landzberg M, Machado RF, et al: Updated clinical classification of pulmonary hypertension. J Am Coll Cardiol 62 (25 Suppl): D34-D41, 2013.

2. Xiong PY, Potus F, Chan W and Archer SL: Models and molecular mechanisms of world health organization group 2 to 4 pulmonary hypertension. Hypertension 71: 34-55, 2018.

3. Shi N and Chen SY: Mechanisms simultaneously regulate smooth muscle proliferation and differentiation. J Biomed Res 28: 40-46, 2014.

4. Masudo K, Suganuma N, Nakayama H, Oshima T, Rino Y, Iwasaki H, Matsuzu K, Sugino K, Ito K, Kondo T, et al: EZH2 overexpression as a useful prognostic marker for aggressive behaviour in thyroid cancer. In Vivo 32: 25-31, 2018.

5. Simon JA and Lange CA: Roles of the EZH2 histone methyltransferase in cancer epigenetics. Mutat Res 647: 21-29, 2008.

6. Chang CJ and Hung MC: The role of EZH2 in tumour progression. Br J Cancer 106: 243-247, 2012.

7. Rao ZY, Cai MY, Yang GF, He LR, Mai SJ, Hua WF, Liao YJ, Deng HX, Chen YC, Guan XY, et al: EZH2 supports ovarian carcinoma cell invasion and/or metastasis via regulation of TGF-beta1 and is a predictor of outcome in ovarian carcinoma patients. Carcinogenesis 31: 1576-1583, 2010.

8. Nephew KP and Huang TH: Epigenetic gene silencing in cancer initiation and progression. Cancer Lett 190: 125-133, 2003.

9. Archer SL, Marsboom G, Kim GH, Zhang HJ, Toth PT, Svensson EC, Dyck JR, Gomberg-Maitland M, Thébaud B, Husain AN, et al: Epigenetic attenuation of mitochondrial superoxide dismutase 2 in pulmonary arterial hypertension: A basis for excessive cell proliferation and a new therapeutic target. Circulation 121: 2661-2671, 2010.
10. Philibert RA, Sears RA, Powers LS, Nash E, Bair T, Gerke AK, Hassan I, Thomas CP, Gross TJ and Monick MM: Coordinated DNA methylation and gene expression changes in smoker alveolar macrophages: Specific effects on VEGF receptor 1 expression. J Leukoc Biol 92: 621-631, 2012.

11. Aljubran SA, Cox R Jr, Tamarapu Parthasarathy P, Kollongod Ramanathan G, Rajanbabu V, Bao H, Mohapatra SS, Lockey R and Kolliputi N: Enhancer of zeste homolog 2 induces pulmonary artery smooth muscle cell proliferation. PLoS One 7: e37712, 2012 .

12. Stenmark KR, Fagan KA and Frid MG: Hypoxia-induced pulmonary vascular remodeling: Cellular and molecular mechanisms. Circ Res 99: 675-691, 2006.

13. Groves BM, Reeves JT, Sutton JR, Wagner PD, Cymerman A, Malconian MK, Rock PB, Young PM and Houston CS: Operation everest II: Elevated high-altitude pulmonary resistance unresponsive to oxygen. J Appl Physiol (1985) 63: 521-530, 1987.

14. Galiè N and Kim NH: Pulmonary microvascular disease in chronic thromboembolic pulmonary hypertension. Proc Am Thorac Soc 3: 571-576, 2006

15. Barst RJ, McGoon M, Torbicki A, Sitbon O, Krowka MJ, Olschewski H and Gaine S: Diagnosis and differential assessment of pulmonary arterial hypertension. J Am Coll Cardiol 43 (12 Suppl S): 40S-47S, 2004

16. Wang Y, Huang X, Leng D, Li J, Wang L, Liang Y, Wang J, Miao R and Jiang T: DNA methylation signatures of pulmonary arterial smooth muscle cells in chronic thromboembolic pulmonary hypertension. Physiol Genomics 50: 313-322, 2018.

17. Ogawa A, Firth AL, Yao W, Madani MM, Kerr KM, Auger WR, Jamieson SW, Thistlethwaite PA and Yuan JX: Inhibition of mTOR attenuates store-operated $\mathrm{Ca}^{2+}$ entry in cells from endarterectomized tissues of patients with chronic thromboembolic pulmonary hypertension. Am J Physiol Lung Cell Mol Physiol 297: L666-L676, 2009.

18. Kanehisa M and Goto S: KEGG: Kyoto encyclopedia of genes and genomes. Nucleic Acids Res 28: 27-30, 2000.

19. Rouillard AD, Gundersen GW, Fernandez NF, Wang Z, Monteiro CD, McDermott MG and Ma'ayan A: The harmonizome: A collection of processed datasets gathered to serve and mine knowledge about genes and proteins. Database (Oxford) 2016: baw100, 2016.

20. Jassal B, Matthews L, Viteri G, Gong C, Lorente P, Fabregat A, Sidiropoulos K, Cook J, Gillespie M, Haw R, et al: The reactome pathway knowledgebase. Nucleic Acids Res 48 (D1): D498-D503, 2020.

21. Xie C, Mao X, Huang J, Ding Y, Wu J, Dong S, Kong L, Gao G, Li CY and Wei L: KOBAS 2.0: A web server for annotation and identification of enriched pathways and diseases. Nucleic Acids Res 39 (Web Server Issue): W316-W322, 2011.

22. Humbert M: Pulmonary arterial hypertension and chronic thromboembolic pulmonary hypertension: Pathophysiology. Eur Respir Rev 19: 59-63, 2010.

23. Czermin B, Melfi R, McCabe D, Seitz V, Imhof A and Pirrotta V: Drosophila enhancer of Zeste/ESC complexes have a histone H3 methyltransferase activity that marks chromosomal Polycomb sites. Cell 111: 185-196, 2002.

24. Bracken AP, Pasini D, Capra M, Prosperini E, Colli E and Helin K: EZH2 is downstream of the pRB-E2F pathway, essential for proliferation and amplified in cancer. EMBO J 22: 5323-5335, 2003.

25. Zhang YX, Li JF, Yang YH, Zhai ZG, Gu S, Liu Y, Miao R, Zhong PP, Wang Y, Huang XX, et al: Renin-angiotensin system regulates pulmonary arterial smooth muscle cell migration in chronic thromboembolic pulmonary hypertension. Am J Physiol Lung Cell Mol Physiol 314: L276-L286, 2018.

26. Wang L, Liu J, Wang W, Qi X, Wang Y, Tian B, Dai H, Wang J, Ning W, Yang T and Wang C: Targeting IL-17 attenuates hypoxia-induced pulmonary hypertension through downregulation of $\beta$-catenin. Thorax 74: 564-578, 2019.

27. Varambally S, Cao Q, Mani RS, Shankar S, Wang X, Ateeq B, Laxman B, Cao X, Jing X, Ramnarayanan K, et al: Genomic loss of microRNA-101 leads to overexpression of histone methyltransferase EZH2 in cancer. Science 322: 1695-1699, 2008.

28. Frid MG, Moiseeva EP and Stenmark KR: Multiple phenotypically distinct smooth muscle cell populations exist in the adult and developing bovine pulmonary arterial media in vivo. Circ Res 75: 669-681, 1994.

29. Pugliese SC, Poth JM, Fini MA, Olschewski A, El Kasmi KC and Stenmark KR: The role of inflammation in hypoxic pulmonary hypertension: From cellular mechanisms to clinical phenotypes. Am J Physiol Lung Cell Mol Physiol 308: L229-L252, 2015. 
30. Rose-John S: Interleukin-6 family cytokines. Cold Spring Harb Perspect Biol 10: a028415, 2018.

31. Karan D, Tawfik O and Dubey S: Expression analysis of inflammasome sensors and implication of NLRP12 inflammasome in prostate cancer. Sci Rep 7: 4378, 2017.

32. Allen IC: Non-inflammasome forming NLRs in inflammation and tumorigenesis. Front Immunol 5: 169, 2014.

33. Hermanns HM: Oncostatin M and interleukin-31: Cytokines, receptors, signal transduction and physiology. Cytokine Growth Factor Rev 26: 545-558, 2015.

34. Miyata M, Ito M, Sasajima T, Ohira H and Kasukawa R: Effect of a serotonin receptor antagonist on interleukin-6-induced pulmonary hypertension in rats. Chest 119: 554-561, 2001.

35. Unver N and McAllister F: IL-6 family cytokines: Key inflammatory mediators as biomarkers and potential therapeutic targets. Cytokine Growth Factor Rev 41: 10-17, 2018.

36. Ye RD and Sun L: Emerging functions of serum amyloid A in inflammation. J Leukoc Biol 98: 923-929, 2015.

37. Helgadottir A, Manolescu A, Thorleifsson G, Gretarsdottir S, Jonsdottir H, Thorsteinsdottir U, Samani NJ, Gudmundsson G, Grant SF, Thorgeirsson G, et al: The gene encoding 5-lipoxygenase activating protein confers risk of myocardial infarction and stroke. Nat Genet 36: 233-239, 2004

38. Malle E, Sodin-Semrl S and Kovacevic A: Serum amyloid A: An acute-phase protein involved in tumour pathogenesis. Cell Mol Life Sci 66: 9-26, 2009.

39. De Buck M, Gouwy M, Wang JM, Van Snick J, Opdenakker G, Struyf S and Van Damme J: Structure and expression of different serum amyloid a (SAA) variants and their concentration-dependent functions during host insults. Curr Med Chem 23: 1725-1755, 2016.

40. Jumeau C, Awad F, Assrawi E, Cobret L, Duquesnoy P, Giurgea I, Valeyre D, Grateau G, Amselem S, Bernaudin JF and Karabina SA: Expression of SAA1, SAA2 and SAA4 genes in human primary monocytes and monocyte-derived macrophages. PLoS One 14: e0217005, 2019.

41. Wang Y, Barbacioru C, Hyland F, Xiao W, Hunkapiller KL, Blake J, Chan F, Gonzalez C, Zhang L and Samaha RR: Large scale real-time PCR validation on gene expression measurements from two commercial long-oligonucleotide microarrays. BMC Genomics 7: 59, 2006.

42. Soon E, Crosby A, Southwood M, Yang P, Tajsic T, Toshner M, Appleby S, Shanahan CM, Bloch KD, Pepke-Zaba J, et al: Bone morphogenetic protein receptor type II deficiency and increased inflammatory cytokine production. A gateway to pulmonary arterial hypertension. Am J Respir Crit Care Med 192: 859-872, 2015.

43. Humbert M, Monti G, Brenot F, Sitbon O, Portier A, Grangeot-Keros L, Duroux P, Galanaud P, Simonneau G and Emilie D: Increased interleukin-1 and interleukin-6 serum concentrations in severe primary pulmonary hypertension. Am J Respir Crit Care Med 151: 1628-1631, 1995.

44. Furuya Y, Satoh T and Kuwana M: Interleukin-6 as a potential therapeutic target for pulmonary arterial hypertension. Int J Rheumatol 2010: 720305, 2010.

45. Yang X, Coriolan D, Murthy V, Schultz K, Golenbock DT and Beasley D: Proinflammatory phenotype of vascular smooth muscle cells: Role of efficient Toll-like receptor 4 signaling. Am J Physiol Heart Circ Physiol 289: H1069-H1076, 2005.
46. Bonnet S, Michelakis ED, Porter CJ, Andrade-Navarro MA, Thébaud B, Bonnet S, Haromy A, Harry G, Moudgil R, McMurtry MS, et al: An abnormal mitochondrial-hypoxia inducible factor-1alpha-Kv channel pathway disrupts oxygen sensing and triggers pulmonary arterial hypertension in fawn hooded rats: Similarities to human pulmonary arterial hypertension. Circulation 113: 2630-2641, 2006.

47. Sutendra G and Michelakis ED: The metabolic basis of pulmonary arterial hypertension. Cell Metab 19: 558-573, 2014.

48. Bonnet S, Archer SL, Allalunis-Turner J, Haromy A, Beaulieu C, Thompson R, Lee CT, Lopaschuk GD, Puttagunta L, Bonnet $\mathrm{S}$, et al: A mitochondria- $\mathrm{K}^{+}$channel axis is suppressed in cancer and its normalization promotes apoptosis and inhibits cancer growth. Cancer Cell 11: 37-51, 2007.

49. Archer SL, Gomberg-Maitland M, Maitland ML, Rich S, Garcia JG and Weir EK: Mitochondrial metabolism, redox signaling, and fusion: A mitochondria-ROS-HIF-1alpha-Kv1.5 $\mathrm{O} 2$-sensing pathway at the intersection of pulmonary hypertension and cancer. Am J Physiol Heart Circ Physiol 294: H570-H578, 2008

50. Cho KA, Suh JW, Lee KH, Kang JL and Woo SY: IL-17 and IL-22 enhance skin inflammation by stimulating the secretion of IL-1 $\beta$ by keratinocytes via the ROS-NLRP3-caspase-1 pathway. Int Immunol 24: 147-158, 2012.

51. Sutendra G, Dromparis P, Bonnet S, Haromy A, McMurtry MS, Bleackley RC and Michelakis ED: Pyruvate dehydrogenase inhibition by the inflammatory cytokine TNF $\alpha$ contributes to the pathogenesis of pulmonary arterial hypertension. J Mol Med (Berl) 89: 771-783, 2011.

52. Yasui $\mathrm{K}$ and Baba A: Therapeutic potential of superoxide dismutase (SOD) for resolution of inflammation. Inflamm Res 55: 359-363, 2006.

53. Rabinovitch $M$, Guignabert $C$, Humbert $M$ and Nicolls $M R$ : Inflammation and immunity in the pathogenesis of pulmonary arterial hypertension. Circ Res 115: 165-175, 2014.

54. Bauer EM, Zheng H, Comhair S, Erzurum S, Billiar TR and Bauer PM: Complement C3 deficiency attenuates chronic hypoxia-induced pulmonary hypertension in mice. PLoS One 6 : e28578, 2011.

55. Zhang J, Zhang Y, Li N, Liu Z, Xiong C, Ni X, Pu Y, Hui R, He J and $\mathrm{Pu}$ J: Potential diagnostic biomarkers in serum of idiopathic pulmonary arterial hypertension. Respir Med 103: 1801-1806, 2009.

56. Langer F, Schramm R, Bauer M, Tscholl D, Kunihara T and Schäfers HJ: Cytokine response to pulmonary thromboendarterectomy. Chest 126: 135-141, 2004.

57. von Haehling S, von Bardeleben RS, Kramm T, Thiermann Y, Niethammer M, Doehner W, Anker SD, Munzel T, Mayer E and Genth-Zotz S: Inflammation in right ventricular dysfunction due to thromboembolic pulmonary hypertension. Int J Cardiol 144: 206-211, 2010.

58. Gu S, Su P, Yan J, Zhang X, An X, Gao J, Xin R and Liu Y: Comparison of gene expression profiles and related pathways in chronic thromboembolic pulmonary hypertension. Int J Mol Med 33: 277-300, 2014.

This work is licensed under a Creative Commons Attribution-NonCommercial-NoDerivatives 4.0 International (CC BY-NC-ND 4.0) License. 